\title{
How images combine meaning: quantum entanglement in visual perception
}

\author{
Jonito Aerts Arguëlles ${ }^{1}$. Sandro Sozzo ${ }^{2}$
}

Published online: 21 January 2020

(c) The Author(s) 2020

\begin{abstract}
Various empirical tests performed on human participants and also by means of search engines on the Web reveal that, whenever the conceptual combination The Animal Acts is considered as a combination of the individual concepts Animal and Acts, the 'Clauser-Horne-Shimony-Holt' version of Bell's inequalities ('CHSH inequality') is violated. We work out in this paper a quantum representation in Hilbert space for a dataset collected on the same combination of concepts using 'Google Images' as search engine, which 'significantly violated' the CHSH inequality. This result proves the existence of non-classical structures in visual perception and strongly indicates the presence of 'quantum entanglement' as an explanation for the meaning connection between the component concepts, also when this meaning connection is expressed through images.
\end{abstract}

Keywords CHSH inequality · Quantum structures · Visual perception · Meaning connection

\section{Introduction}

Among the quantum effects recently identified within the quantum cognition research program (Aerts 2009; Aerts et al. 2013a,b, 2018; Busemeyer and Bruza 2012; Dalla Chiara et al. 2015a, b; Khrennikov 2010; Melucci 2015), quantum entanglement is certainly one of the most important and intriguing ones, with the additional advantage that its quantum fingerprint can directly be tested experimentally (Aerts and Sozzo 2011; Bruza et al. 2009, 2015; Gronchi and Strambini 2017; Beltran and Geriente 2019).

It is indeed well known from the research on the foundations of quantum theory that entanglement is responsible for quantum entities to exhibit non-classical correlations which are actualized at a distance and can be tested experimentally since they violate Bell's inequalities (Bell 1987), includ-

Communicated by Federico Holik.

Sandro Sozzo

ss831@le.ac.uk

Jonito Aerts Arguëlles

jonitoarguelles@gmail.com

1 Center Leo Apostel for Interdisciplinary Studies, Free University of Brussels (VUB), Krijgskundestraat 33, 1160 Brussels, Belgium

2 School of Business and IQSCS, University of Leicester, University Road, Leicester LE17RH, UK ing one of its versions, the Clauser-Horne-Shimony-Holt (CHSH) inequality (Clauser et al. 1969). These non-classical correlations are nowadays widely exploited in quantum technologies, which use the peculiarities of entangled states, and also entangled measurements, to encrypt data, process quantum information, simulate quantum computation tasks, teleport unknown quantum states, etc.

Recently, situations of composite entities violating the CHSH inequality were identified in human cognition, precisely in the way individual concepts are combined in the human mind through meaning.

Our research team has been engaged for two decades in theoretical and experimental studies of entanglement outside the microscopic domain (Aerts et al. 2000).

More specifically, we performed a psychological test asking 81 human participants to choose in a questionnaire the exemplars, e.g., Bear Growls, Tiger Snorts, Cat Meows, etc., that they elected as 'good examples' of the conceptual combination The Animal Acts, considered as a combination of the individual concepts Animal and Acts. We found that the empirical probabilities violated the $\mathrm{CHSH}$ inequality by a numerical value of 2.4197, which is close to the CHSH violation found in Bell tests on quantum particles (Aerts and Sozzo 2011). This investigation showed that individual conceptual entities, like Animal and Acts, exhibit a meaning connection, whenever they combine to form the bipartite conceptual 
entity The Animal Acts, which cannot be explained in terms of classical structures.

The study of 2011 left open the question of whether the non-classical correlations of Animal and Acts are genuinely quantum, though strong indications were provided in this direction. The loop was closed in 2014, when we worked out a quantum model in Hilbert space of the data in Aerts and Sozzo (2011). However, a somewhat unexpected result was obtained: the meaning connection between Animal and Acts can indeed be explained as due to quantum entanglement, but the latter is even stronger than the one that was originally found in Bell tests in quantum physics, as entanglement is present in both the states of the composite entity The Animal Acts and the joint measurements that are performed on this composite entity and enter the CHSH inequality. In other words, whenever we ask a sample of participants which exemplar among Horse Growls, Horse Whinnies, Bear Growls and Bear Whinnies is a good example of The Animal Acts, this operation cannot be generally decomposed into an operation in which we separately ask which exemplar between Horse and Bear is a good example of Animal 'and' which exemplar between Bear and Whinnies is a good example of Acts (Aerts and Sozzo 2014).

The outcome above was a priori unexpected, but should not surprise too much: indeed, it is true that, for example, Horse Whinnies is just a juxtaposition of the words 'horse' and 'whinnies', but the latter words also express concepts which are connected by meaning. Hence, if we accept the idea of considering concepts as 'entities in a specific state' and not as 'containers of objects', then this meaning connection is exactly captured by entangled states. And, it is a standard quantum result that any measurement having entangled states as outcome states is an entangled measurement.

Our research team provided additional evidence of entanglement in concept combination in 2018, when we discovered that the CHSH inequality is also violated in a Web test where the exemplars of The Animal Acts are 'chosen' in document retrieval operations using specific corpuses of documents, like 'Google Books' (Beltran and Geriente 2019). Also here, however, an unanticipated result was attained: the $\mathrm{CHSH}$ inequality is violated by an amount that exceeds the famous 'Cirel'son bound' for genuinely quantum violations (Cirel'son 1980)_for example, the CHSH factor is equal to 3.4058 , definitely higher than the bound of $2 \sqrt{2}=2.8284$ predicted by quantum theory for entangled states and product measurements. We can again explain this result by assuming that meaning connection is created by entanglement and that this entanglement is present in both states and measurements. In fact, we proved in a quantum Hilbert space representation of the Web data that exemplars that are high-meaning connected, like Bear Growls, or Tiger Snorts, are described by entangled states, while exemplars that are low-meaning con- nected, like Horse Whinnies or Bear Meows, are described by product states (Aerts et al. 2019a).

In the present paper, we further extend our research on entanglement in the combination of meaning entities, elaborating a quantum representation in Hilbert space for the empirical data collected by one of us in a test on visual perception where the probabilistic data on The Animal Acts to be inserted into the $\mathrm{CHSH}$ inequality are obtained by counting the images retrieved using 'Google Images' as search engine (Aerts Arguëlles 2018).

We firstly review the empirical procedures which were followed in the 'Google Images' test, observing that it significantly violates the $\mathrm{CHSH}$ inequality, hence showing that non-classical structures are present in the combination of entities of meaning (Sect.2), which in this case are images. Then, we elaborate a Hilbert space representation of the data, using the formal procedures employed in Aerts and Sozzo (2014) and Aerts et al. (2019a). We prove that the violation of the CHSH inequality in the 'Google Images' test can be explained by assuming that a stronger form of entanglement is responsible for the identified meaning connection, in which both the state and the joint measurements are entangled (Sect. 3). We finally compare the results obtained in the psychological test with those obtained in the visual perception test (Sect. 4).

The result obtained in this paper provides further theoretical and empirical support to the assumption that 'quantum entanglement captures both conceptually and mathematically the meaning connection existing between conceptual entities whenever these combine'. This research fits a general unifying and explanatory perspective on the phenomenon of entanglement in bipartite systems, as it manifests in microand macrophysical systems, as well as in human cognition, we have recently developed (Aerts et al. 2019b).

\section{Violation of the CHSH inequality in visual perception}

In Aerts Arguëlles (2018), the conceptual combination The Animal Acts with different of its exemplars, e.g., The Tiger Growls, The Cat Meows, etc. was investigated as expressed by pictures. 'Google Images' were used to calculate the relative frequencies of appearance of the pictures expressing the exemplars of The Animal Acts, and they were interpreted as 'probabilities of appearance' and inserted into the CHSH version of Bell's inequalities (Clauser et al. 1969). The significant violation of the $\mathrm{CHSH}$ inequality that resulted constitutes a strong indication of the presence of 'quantum entanglement in visual perception'.

This violation shows a remarkable similarity with the violation of the CHSH inequality in a psychological test on human participants (Aerts and Sozzo 2011) and a Web test on 
corpuses of documents (Aerts et al. 2019a), where the same combination of concepts The Animal Acts and the same sets of exemplars were used. This result hence adds to the evidence for the robustness of the presence of the entanglement identified by the violation of this CHSH inequality.

To calculate the term $E\left(A^{\prime}, B^{\prime}\right)+E\left(A, B^{\prime}\right)+E\left(A^{\prime}, B\right)-$ $E(A, B)$ that appears in the CHSH inequality

$$
-2 \leq E\left(A^{\prime}, B^{\prime}\right)+E\left(A, B^{\prime}\right)+E\left(A^{\prime}, B\right)-E(A, B) \leq 2,
$$

we traditionally consider four experiments $A B, A B^{\prime}, A^{\prime} B$, and $A^{\prime} B^{\prime}$, each of them consisting in a joint experiment. For example, $A B$ is the experiment consisting in jointly performing experiments on the concepts $A$ and $B$. In our case, the concepts and their combinations and their exemplars are expressed in pictures, which means that our experiments consist actually of applying the 'Google Images' tool as a search engine for the different exemplars of the considered combinations of concepts and following specific procedures, which we specify in the following, to calculate the probabilities of occurrence of the different outcomes. The quantities $E(A, B), E\left(A, B^{\prime}\right), E\left(A^{\prime}, B\right)$, and $E\left(A^{\prime}, B^{\prime}\right)$ are the expected values of these experiments and will be calculated by gathering data on $A B, A B^{\prime}, A^{\prime} B$, and $A^{\prime} B^{\prime}$.

Let us start by experiment $A B$. Two exemplars Horse and Bear are considered for the concept Animal, and two exemplars Growls and Whinnies are considered for the concept Acts. The four combinations The Horse Growls, The Horse Whinnies, The Bear Growls, and The Bear Whinnies, where each of them is an exemplar of The Animal Acts, are considered for the experiment $A B$. These four combinations play the role of the four outcomes of the experiment $A B$, and outcome probabilities are attributed to them following the procedure we will specify now. We make a search in 'Google Images' on 'horse growls', entering the two words in the ordered sequence in the 'Google' bar, finding 400 images by one hit of 'Google Images'. We then carefully inspect each of the 400 images and count the number of them on which we see a horse that growls. This of course contains some subjective appreciation of the images, because we only have access to visual information. Proceeding in this way in Aerts Arguëlles (2018), we could conclude that four images over 400 possibly showed a horse that is growling. Next, we divide the number of images showing a horse that growls by the total number of images inspected. For the data collected in Aerts Arguëlles (2018), this gave $\mathscr{P}\left(A_{1}, B_{1}\right)=\frac{4}{400}=0.01$.

Let us come to the search in 'Google Images' on 'horse whinnies'. We proceed in analogous way as explained for 'horse growls'. For the data collected in Aerts Arguëlles (2018), then 391 images showed up as search results. A careful verification of each image gave the count of 39 of them showing a horse that whinnies. Hence, the corresponding probability is $\mathscr{P}\left(A_{1}, B_{2}\right)=\frac{39}{391}=0.0997$. The search in
'Google Images' for 'bear growls' gave 380 images, while 142 of them showed a bear that growls. Hence, the corresponding probability is $\mathscr{P}\left(A_{2}, B_{1}\right)=\frac{142}{380}=0.3737$. Finally, the search in 'Google Images' on 'bear whinnies' gave 389 images, while two of them could be interpreted as a bear that whinnies. This gives a probability $\mathscr{P}\left(A_{2}, B_{2}\right)=$ $\frac{2}{389}=0.0050$.

In the experiment $A B$, we thus obtained in Aerts Arguëlles (2018) four probabilities $\mathscr{P}\left(A_{1}, B_{1}\right), \mathscr{P}\left(A_{1}, B_{2}\right), \mathscr{P}\left(A_{2}\right.$, $\left.B_{1}\right)$, and $\mathscr{P}\left(A_{2}, B_{2}\right)$, connected with the chance to find a horse that growls, a horse that whinnies, a bear that growls, and a bear that whinnies, respectively, in a search with 'Google Images' explained above. However, to formulate the CHSH inequality, we need to further normalize these quantities. More specifically, we need estimations of "what is the probability that one of the four will appear if we know that at least one of them is appearing'. We can obtain these renormalized probabilities by dividing each of the now calculated probability $\mathscr{P}(\cdot)$ by their sum:

$$
\begin{aligned}
& \mathscr{S}(A, B)=\mathscr{P}\left(A_{1}, B_{1}\right)+\mathscr{P}\left(A_{1}, B_{2}\right) \\
& +\mathscr{P}\left(A_{2}, B_{1}\right)+\mathscr{P}\left(A_{2}, B_{2}\right)=0.4884
\end{aligned}
$$

This gives:

$$
\begin{aligned}
P\left(A_{1}, B_{1}\right) & =\frac{\mathscr{P}\left(A_{1}, B_{1}\right)}{\mathscr{S}(A, B)}=0.0205 \\
P\left(A_{1}, B_{2}\right) & =\frac{\mathscr{P}\left(A_{1}, B_{2}\right)}{\mathscr{S}(A, B)}=0.2042 \\
P\left(A_{2}, B_{1}\right) & =\frac{\mathscr{P}\left(A_{2}, B_{1}\right)}{\mathscr{S}(A, B)}=0.7651 \\
P\left(A_{2}, B_{2}\right) & =\frac{\mathscr{P}\left(A_{2}, B_{2}\right)}{\mathscr{S}(A, B)}=0.0103
\end{aligned}
$$

The corresponding expectation value $E(A, B)$ is then

$$
\begin{aligned}
& E(A, B)=P\left(A_{1}, B_{1}\right)-P\left(A_{1}, B_{2}\right) \\
& \quad-P\left(A_{2}, B_{1}\right)+P\left(A_{2}, B_{1}\right)=-0.9385
\end{aligned}
$$

The assumption underlying the above expectation value is that the choice Horse for Animal is given the value +1 , while the choice Bear for Animal is given the value -1. Similarly, the choice Growls for Acts is given the value +1 , and the choice Whinnies for Acts which is given the value -1 . Then, combining these values, we obtain that the choice The Horse Growls is associated with the value +1 , obtained by multiplying the value +1 for Horse with the value +1 for Growls. Similarly, the choice The Horse Whinnies is -1 (multiplying +1 by -1 ), the choice The Bear Growls is -1 (multiplying -1 by 1 ) and the choice The Bear Whinnies is +1 (multiplying -1 by -1$)$. Then, $E(A, B)$ is the 'expected value' given by the probabilities $P\left(A_{1}, B_{1}\right), P\left(A_{1}, B_{2}\right), P\left(A_{2}, B_{1}\right)$, and 
$P\left(A_{2}, B_{1}\right)$ of each of these values. $E(A, B)=-0.9385$ particularly means that there is a strong anti-correlation, and indeed, Horse anti-correlates with Growls and Bear anticorrelates with Whinnies.

To define the three remaining experiments $A B^{\prime}, A^{\prime} B$, and $A^{\prime} B^{\prime}$ that are needed to calculate the CHSH inequality term, we consider two different exemplars of Animal, as well as two different exemplars of Acts, namely Tiger and Cat, and Snorts and Meows, respectively. In the experiment $A B^{\prime}$, the exemplars Horse and Bear of Animal are combined with the exemplars Snorts and Meows of Acts; in $A^{\prime} B$, the exemplars Tiger and Cat of Animal are combined with the exemplars Growls and Whinnies of Acts; in $A^{\prime} B^{\prime}$, the exemplars Tiger and Cat of Animal are combined with the exemplars Snorts and Meows of Acts.

Concretely, in the experiment $A B^{\prime}$, we made a 'Google Images' search of 'horse snorts', obtaining 407 images of which 41 show a snorting horse, a search of 'horse meows', obtaining 403 images of which four show a meowing horse, a search of 'bear snorts', obtaining 385 images of which 23 show a snorting bear and a search of 'bear meows', obtaining 405 images of which five show a meowing bear. This gives, using the symbols above, $\mathscr{P}\left(A_{1}, B_{1}^{\prime}\right)=$ $0.1007, \mathscr{P}\left(A_{1}, B_{2}^{\prime}\right)=0.0099, \mathscr{P}\left(A_{2}, B_{1}^{\prime}\right)=0.0597$, and $\mathscr{P}\left(A_{2}, B_{2}^{\prime}\right)=0.0123$, and hence

$$
\begin{gathered}
\mathscr{S}\left(A, B^{\prime}\right)=\mathscr{P}\left(A_{1}, B_{1}^{\prime}\right)+\mathscr{P}\left(A_{1}, B_{2}^{\prime}\right) \\
\quad+\mathscr{P}\left(A_{2}, B_{1}^{\prime}\right)+\mathscr{P}\left(A_{2}, B_{2}^{\prime}\right)=0.1827 \\
P\left(A_{1}, B_{1}^{\prime}\right)=\frac{\mathscr{P}\left(A_{1}, B_{1}^{\prime}\right)}{\mathscr{S}\left(A, B^{\prime}\right)}=0.5512 \\
P\left(A_{1}, B_{2}^{\prime}\right)=\frac{\mathscr{P}\left(A_{1}, B_{2}^{\prime}\right)}{\mathscr{S}\left(A, B^{\prime}\right)}=0.0543 \\
P\left(A_{2}, B_{1}^{\prime}\right)=\frac{\mathscr{P}\left(A_{2}, B_{1}^{\prime}\right)}{\mathscr{S}\left(A, B^{\prime}\right)}=0.3269 \\
P\left(A_{2}, B_{2}^{\prime}\right)=\frac{\mathscr{P}\left(A_{2}, B_{2}^{\prime}\right)}{\mathscr{S}\left(A, B^{\prime}\right)}=0.0676 \\
E\left(A, B^{\prime}\right)=P\left(A_{1}, B_{1}^{\prime}\right)-P\left(A_{1}, B_{2}^{\prime}\right) \\
\quad-P\left(A_{2}, B_{1}^{\prime}\right)+P\left(A_{2}, B_{1}^{\prime}\right)=0.2376
\end{gathered}
$$

In the experiment $A^{\prime} B$, we made a 'Google Images' search of 'tiger growls', obtaining 399 images of which 219 show a growling tiger, a search of 'tiger whinnies', obtaining 402 images of which three show a whinnying tiger, a search of "cat growls', obtaining 405 images of which 78 show a growling cat and a search of 'cat whinnies', obtaining 402 images of which one shows a whinnying cat. This gives $\mathscr{P}\left(A_{1}^{\prime}, B_{1}\right)=$ $0.5489, \mathscr{P}\left(A_{1}^{\prime}, B_{2}\right)=0.0075, \mathscr{P}\left(A_{2}^{\prime}, B_{1}\right)=0.1926$, and $\mathscr{P}\left(A_{2}^{\prime}, B_{2}\right)=0.0025$, and hence

$$
\begin{aligned}
& \mathscr{S}\left(A^{\prime}, B\right)=\mathscr{P}\left(A_{1}^{\prime}, B_{1}\right)+\mathscr{P}\left(A_{1}^{\prime}, B_{2}\right) \\
& +\mathscr{P}\left(A_{2}^{\prime}, B_{1}\right)+\mathscr{P}\left(A_{2}^{\prime}, B_{2}\right)=0.7514
\end{aligned}
$$

$$
\begin{aligned}
& P\left(A_{1}^{\prime}, B_{1}\right)=\frac{\mathscr{P}\left(A_{1}^{\prime}, B_{1}\right)}{\mathscr{S}\left(A^{\prime}, B\right)}=0.7305 \\
& P\left(A_{1}^{\prime}, B_{2}\right)=\frac{\mathscr{P}\left(A_{1}^{\prime}, B_{2}\right)}{\mathscr{S}\left(A^{\prime}, B\right)}=0.0099 \\
& P\left(A_{2}^{\prime}, B_{1}\right)=\frac{\mathscr{P}\left(A_{2}^{\prime}, B_{1}\right)}{\mathscr{S}\left(A^{\prime}, B\right)}=0.2563 \\
& P\left(A_{2}^{\prime}, B_{2}\right)=\frac{\mathscr{P}\left(A_{2}^{\prime}, B_{2}\right)}{\mathscr{S}\left(A^{\prime}, B\right)}=0.0033 \\
& E\left(A^{\prime}, B\right)=P\left(A_{1}^{\prime}, B_{1}\right)-P\left(A_{1}^{\prime}, B_{2}\right) \\
& \quad-P\left(A_{2}^{\prime}, B_{1}\right)+P\left(A_{2}^{\prime}, B_{1}\right)=0.4675
\end{aligned}
$$

Finally, in the experiment $A^{\prime} B^{\prime}$, we made a 'Google Images' search of 'tiger snorts', obtaining 400 images of which 30 show a snorting tiger, a search of 'tiger meows', obtaining 403 images of which ten show a meowing tiger, a search of 'cat snorts', obtaining 392 images of which 15 show a snorting cat, and a search of 'cat meows', obtaining 399 images of which 161 show a meowing cat. This gives $\mathscr{P}\left(A_{1}^{\prime}, B_{1}^{\prime}\right)=$ $0.075, \mathscr{P}\left(A_{1}^{\prime}, B_{2}^{\prime}\right)=0.0248, \mathscr{P}\left(A_{2}^{\prime}, B_{1}^{\prime}\right)=0.0383$, and $\mathscr{P}\left(A_{2}^{\prime}, B_{2}^{\prime}\right)=0.4035$, and hence

$$
\begin{gathered}
\mathscr{S}\left(A^{\prime}, B^{\prime}\right)=\mathscr{P}\left(A_{1}^{\prime}, B_{1}^{\prime}\right)+\mathscr{P}\left(A_{1}^{\prime}, B_{2}^{\prime}\right) \\
\quad+\mathscr{P}\left(A_{2}^{\prime}, B_{1}^{\prime}\right)+\mathscr{P}\left(A_{2}^{\prime}, B_{2}^{\prime}\right)=0.5416 \\
P\left(A_{1}^{\prime}, B_{1}^{\prime}\right)=\frac{\mathscr{P}\left(A_{1}^{\prime}, B_{1}^{\prime}\right)}{\mathscr{S}\left(A^{\prime}, B^{\prime}\right)}=0.1385 \\
P\left(A_{1}^{\prime}, B_{2}^{\prime}\right)=\frac{\mathscr{P}\left(A_{1}^{\prime}, B_{2}^{\prime}\right)}{\mathscr{S}\left(A^{\prime}, B^{\prime}\right)}=0.0458 \\
P\left(A_{2}^{\prime}, B_{1}^{\prime}\right)=\frac{\mathscr{P}\left(A_{2}^{\prime}, B_{1}^{\prime}\right)}{\mathscr{S}\left(A^{\prime}, B^{\prime}\right)}=0.0707 \\
P\left(A_{2}^{\prime}, B_{2}^{\prime}\right)=\frac{\mathscr{P}\left(A_{2}^{\prime}, B_{2}^{\prime}\right)}{\mathscr{S}\left(A^{\prime}, B^{\prime}\right)}=0.7450 \\
E\left(A^{\prime}, B^{\prime}\right)=P\left(A_{1}^{\prime}, B_{1}^{\prime}\right)-P\left(A_{1}^{\prime}, B_{2}^{\prime}\right) \\
-P\left(A_{2}^{\prime}, B_{1}^{\prime}\right)+P\left(A_{2}^{\prime}, B_{1}^{\prime}\right)=0.7671
\end{gathered}
$$

The results above are reported in Table 1 .

We have now all available empirical data and their corresponding calculations of probabilities and expectation values to calculate the term of the $\mathrm{CHSH}$ inequality, which gives

$E\left(A^{\prime}, B^{\prime}\right)+E\left(A, B^{\prime}\right)+E\left(A^{\prime}, B\right)-E(A, B)=2.4107$

As we can see, the CHSH inequality (1) is manifestly violated. In addition, the numerical amount of the violation is very close to the amount of the CHSH inequality violation we found in the psychological test (Aerts and Sozzo 2011). This result strongly points toward non-classical structure in the representation of visual images.

In the next section, we will work out a quantum representation in Hilbert space of the data reported in this section, 
Table 1 Data collected in the experiments on entanglement in the Web test using 'Google Images' as source (Aerts Arguëlles 2018)

\begin{tabular}{lllll}
\hline Experiment $A B$ & 'horse growls' & 'horse whinnies' & 'bear growls' & 'bear whinnies' \\
& $\mu\left(A_{1}, B_{1}\right)=0.0205$ & $P\left(A_{1}, B_{2}\right)=0.2042$ & $\mu\left(A_{2}, B_{1}\right)=0.7651$ & $\mu\left(A_{2}, B_{2}\right)=0.0103$ \\
Experiment $A B^{\prime}$ & 'horse snorts' & 'horse meows' & 'bear snorts' & 'bear meows' \\
& $\mu\left(A_{1}, B_{1}^{\prime}\right)=0.5512$ & $\mu\left(A_{1}, B_{2}^{\prime}\right)=0.0543$ & $\mu\left(A_{2}, B_{1}^{\prime}\right)=0.3269$ & $\mu\left(A_{2}, B_{2}^{\prime}\right)=0.0676$ \\
Experiment $A^{\prime} B$ & 'tiger growls' & 'tiger whinnies' & 'cat growls' & 'cat whinnies' \\
& $\mu\left(A_{1}^{\prime}, B_{1}\right)=0.7305$ & $\mu\left(A_{1}^{\prime}, B_{2}\right)=0.0099$ & $\mu\left(A_{2}^{\prime}, B_{1}\right)=0.2563$ & $\mu\left(A_{2}^{\prime}, B_{2}\right)=0.0033$ \\
Experiment $A^{\prime} B^{\prime}$ & 'tiger snorts' & 'tiger meows' & 'cat snorts' & 'cat meows' \\
& $\mu\left(A_{1}^{\prime}, B_{1}^{\prime}\right)=0.1385$ & $\mu\left(A_{1}^{\prime}, B_{2}^{\prime}\right)=0.0458$ & $\mu\left(A_{2}^{\prime}, B_{1}^{\prime}\right)=0.0707$ & $\mu\left(A_{2}^{\prime}, B_{2}^{\prime}\right)=0.7450$ \\
& & & &
\end{tabular}

which shows that 'quantum entanglement provides a natural unifying explanation for the meaning connections identified in all these kinds of tests'.

\section{Quantum modeling of data on composition of images}

In the Hilbert space representation of the data in Sect. 2, we follow the formal techniques we developed in Aerts and Sozzo (2014) and applied in Aerts et al. (2019a). As mentioned in Sect. 1 and shown in Aerts and Sozzo (2014), the way states are prepared and measurements are performed in The Animal Acts entails that not only the state of the conceptual entity The Animal Acts is entangled, but also the four measurements are generally entangled.

We denote by $p$ the state of the composed conceptual entity The Animal Acts, made up of the individual conceptual entities Animal and Acts.

Experiment $A B$ corresponds to a joint measurement $e_{A B}$ performed on the composite entity The Animal Acts with four possible outcomes $\lambda_{H G}, \lambda_{B W}$ (which are chosen equal to +1 ), $\lambda_{H W}$, and $\lambda_{B G}$ (which are chosen equal to -1 ), and four outcome states $p_{H G}, p_{B W}, p_{H W}$, and $p_{B G}$, describing the situation of The Animal Acts once 'horse growls', 'bear whinnies', 'horse whinnies', and 'bear growls', respectively, are considered in $A B$ using 'Google Images'. Let us denote by $P_{p}(H G), P_{p}(B W), P_{p}(H W)$, and $P_{p}(B G)$ the probability that the outcome $\lambda_{H G}, \lambda_{B W}, \lambda_{H W}$, and $\lambda_{B G}$, respectively, is obtained when The Animal Acts is in the state $p$ and the measurement $e_{A B}$ is performed. ${ }^{1}$

Experiment $A B^{\prime}$ corresponds to a joint measurement $e_{A B^{\prime}}$ on the composite conceptual entity The Animal Acts with four possible outcomes $\lambda_{H S}, \lambda_{B M}$ (which are chosen equal to +1 ), $\lambda_{H S}$, and $\lambda_{B M}$ (which are chosen equal to -1 ), and four outcome states $p_{H S}, p_{B M}, p_{H M}$, and $p_{B S}$, describing the situation of The Animal Acts once 'horse snorts', 'bear

\footnotetext{
${ }^{1}$ For $A B$, the appearance numbers in Table 1, Sect. 2, are an estimation of the probabilities that a given outcome is obtained. The same remark applies to $A B^{\prime}, A^{\prime} B$, and $A^{\prime} B^{\prime}$.
}

meows', 'horse meows', and 'bear snorts', respectively, are considered in $A B^{\prime}$ using 'Google Images'. Let us denote by $P_{p}(H S), P_{p}(B M), P_{p}(H M)$, and $P_{p}(B S)$ the probability that the outcome $\lambda_{H S}, \lambda_{B M}, \lambda_{H M}$, and $\lambda_{B S}$, respectively, is obtained when The Animal Acts is in the state $p$ and the measurement $e_{A B^{\prime}}$ is performed.

Experiment $A^{\prime} B$ corresponds to a joint measurement $e_{A^{\prime} B}$ on the composite conceptual entity The Animal Acts with four possible outcomes $\lambda_{T G}, \lambda_{C W}$ (which are chosen equal to +1 ), $\lambda_{T W}$, and $\lambda_{C G}$ (which are chosen equal to -1 ), and four outcome states $p_{T G}, p_{C W}, p_{T W}$, and $p_{C G}$, describing the situation of The Animal Acts once 'tiger growls', 'cat whinnies', 'tiger whinnies', and 'cat growls', respectively, are considered in $A^{\prime} B$ using 'Google Images'. Let us denote by $P_{p}(T G), P_{p}(C W), P_{p}(T W)$, and $P_{p}(C G)$ the probability that the outcome $\lambda_{T G}, \lambda_{C W}, \lambda_{T W}$, and $\lambda_{C G}$, respectively, is obtained when The Animal Acts is in the state $p$ and the measurement $e_{A^{\prime} B}$ is performed.

Experiment $A^{\prime} B^{\prime}$ corresponds to a joint measurement $e_{A^{\prime} B^{\prime}}$ on the composite conceptual entity The Animal Acts with four possible outcomes $\lambda_{T S}, \lambda_{C M}$ (which are chosen equal to +1$), \lambda_{T M}$, and $\lambda_{C S}$ (which are chosen equal to -1 ), and four outcome states $p_{T S}, p_{C M}, p_{T M}$, and $p_{C S}$, describing the situation of The Animal Acts once 'tiger snorts', 'meows', 'tiger meows', and 'cat snorts', respectively, are considered in $A^{\prime} B^{\prime}$ using 'Google Images'. Let us denote by $P_{p}(T S), P_{p}(C M), P_{p}(T M)$, and $P_{p}(C S)$ the probability that the outcome $\lambda_{T S}, \lambda_{C M}, \lambda_{T M}$, and $\lambda_{C S}$, respectively, is obtained when The Animal Acts is in the state $p$ and the measurement $e_{A^{\prime} B^{\prime}}$ is performed.

Before coming to the mathematical representation, let us preliminarily observe that the violation of the $\mathrm{CHSH}$ inequality indicates that the concepts Animal and Acts are connected by meaning in the combination The Animal Acts. In addition, they are connected in a non-classical way, which suggests that meaning-connected concepts, like The Animal Acts, should be described by entangled states. Furthermore, the joint measurements $e_{A B}, e_{A B^{\prime}}, e_{A^{\prime} B}$, and $e_{A^{\prime} B^{\prime}}$ correspond to operations performed on the overall entity The Animal Acts rather than to operations separately performed on the individual entities Animal and Acts. As such, their outcome states, 
e.g., Horse Whinnies or Tiger Growls, being again meaningconnected combinations of concepts, should be described by entangled states. This entails that the joint measurement $e_{A B}$ (similarly, $e_{A B^{\prime}}, e_{A^{\prime} B}$, and $e_{A^{\prime} B^{\prime}}$ ) on The Animal Acts cannot be generally decomposed into a submeasurement $e_{A}$ on Animal and a submeasurement $e_{B}$ on Acts, though, e.g., Horse Whinnies is syntactically formed by juxtaposing the words 'horse' and 'whinnies', because the concepts Horse and Whinnies are connected by meaning.

Each joint measurement has four distinct outcomes; hence, we can now associate the composed conceptual entity The Animal Acts with the Hilbert space $\mathbb{C}^{4}$ of all 4-tuples of complex numbers. The Hilbert space $\mathbb{C}^{4}$ is canonically isomorphic to the tensor product Hilbert space $\mathbb{C}^{2} \otimes \mathbb{C}^{2}$, where $\mathbb{C}^{2}$ is the complex Hilbert space of all 2-tuples of complex numbers. Let $\{(1,0,0,0),(0,1,0,0),(0,0,1,0),(0,0,0,1)\}$ be the canonical ON basis of $\mathbb{C}^{4}$, which is canonically isomorphic to the ON basis $\{(1,0) \otimes(1,0),(1,0) \otimes(0,1),(0,1) \otimes$ $(1,0),(0,1) \otimes(0,1)\}$ of $\mathbb{C}^{2} \otimes \mathbb{C}^{2}$.

In the canonical ON basis of $\mathbb{C}^{4}$, the initial state $p$ of The Animal Acts is represented by the unit vector $|p\rangle=$ $\left(a e^{i \alpha}, b e^{i \beta}, c e^{i \gamma}, d e^{i \delta}\right)$, where $a, b, c, d$ are nonnegative real numbers such that $a^{2}+b^{2}+c^{2}+d^{2}=1$ and $\alpha, \beta, \gamma, \delta$ are real numbers. The unit vector $|p\rangle$ represents a product state if and only if $a d e^{i(\alpha+\delta)}-b c e^{i(\beta+\gamma)}=0$. Otherwise, $p$ represents an entangled state. However, symmetry reasons suggest us to represent the initial state of the combined concept The Animal Acts by the unit vector

$|p\rangle=\frac{1}{\sqrt{2}}(0,1,-1,0)$

Coming to joint measurements, the measurement $e_{A B}$ has four outcomes $\lambda_{H G}, \lambda_{H W}, \lambda_{B G}$, and $\lambda_{B W}$, corresponding to Horse Growls, Bear Whinnies, Bear Growls, and Bear Whinnies, and four outcome states $p_{H G}, p_{H W}, p_{B G}$, and $p_{B W}$, respectively. The measurement $e_{A B}$ is thus represented by the self-adjoint operator

$$
\begin{gathered}
\mathscr{E}_{A B}=\left|p_{H G}\right\rangle\left\langle p_{H G}|+| p_{B W}\right\rangle\left\langle p_{B W}\right| \\
-\left|p_{H W}\right\rangle\left\langle p_{H W}|-| p_{B G}\right\rangle\left\langle p_{B G}\right|
\end{gathered}
$$

or, equivalently, by the spectral family $\left\{\left|p_{H G}\right\rangle\left\langle p_{H G}|,| p_{H W}\right\rangle\right.$ $\left.\left\langle p_{H W}|,| p_{B G}\right\rangle\left\langle p_{B G}|,| p_{B W}\right\rangle\left\langle p_{B W}\right|\right\}$, such that the outcome states $p_{H G}, p_{H W}, p_{B G}$, and $p_{B W}$ are represented by the eigenvectors of $\mathscr{E}_{A B}$

$$
\begin{aligned}
\left|p_{H G}\right\rangle & =e^{i \Theta_{H G}}\left(a_{H G}, b_{H G}, c_{H G}, d_{H G}\right) \\
\left|p_{H W}\right\rangle & =e^{i \Theta_{H W}}\left(a_{H W}, b_{H W}, c_{H W}, d_{H W}\right) \\
\left|p_{B G}\right\rangle & =e^{i \Theta_{B G}}\left(a_{B G}, b_{B G}, c_{B G}, d_{B G}\right) \\
\left|p_{B W}\right\rangle & =e^{i \Theta_{B W}}\left(a_{B W}, b_{B W}, c_{B W}, d_{B W}\right),
\end{aligned}
$$

respectively. In (29)-(32), we are assuming that the phases of the complex numbers are the same across the various $\mathbb{C}^{4}$ components of the unit vectors, for the sake of simplicity. Moreover, $a_{i j}, b_{i j}, c_{i j}, d_{i j}, i=H, B, j=B, W$, are nonnegative real numbers and $e^{i \Theta_{H G}}, e^{i \Theta_{H W}}, e^{i \Theta_{B G}}$, and $e^{i \Theta_{B W}}$ are real numbers. The self-adjoint operator $\mathscr{E}_{A B}$ represents a product measurement if and only if all unit vectors in (29)(32) represent product states. Otherwise, $e_{A B}$ is an entangled measurement.

To determine the unit vectors in (29)-(32) that satisfy empirical data, we need to impose the following conditions:

(i) the vectors in (29)-(32) have to be unitary;

(ii) the vectors in (29)-(32) have to be mutually orthogonal;

(iii) the empirically determined probabilities of appearance in Table 1, Sect. 2, have to be calculated through the Born rule of quantum probability.

Let us then come to the representation of the other joint measurements. The measurements $e_{A B^{\prime}}, e_{A^{\prime} B}$, and $e_{A^{\prime} B^{\prime}}$ are, respectively, represented by the self-adjoint operators

$$
\begin{aligned}
\mathscr{E}_{A B^{\prime}}= & \left|p_{H S}\right\rangle\left\langle p_{H S}|+| p_{B M}\right\rangle\left\langle p_{B M}\right| \\
& -\left|p_{H M}\right\rangle\left\langle p_{H M}|-| p_{B S}\right\rangle\left\langle p_{B S}\right| \\
\mathscr{E}_{A^{\prime} B}= & \left|p_{T G}\right\rangle\left\langle p_{T G}|+| p_{C W}\right\rangle\left\langle p_{C W}\right| \\
& -\left|p_{T W}\right\rangle\left\langle p_{T W}|-| p_{C G}\right\rangle\left\langle p_{C G}\right| \\
\mathscr{E}_{A^{\prime} B^{\prime}}= & \left|p_{T S}\right\rangle\left\langle p_{T S}|+| p_{C M}\right\rangle\left\langle p_{C M}\right| \\
& -\left|p_{T M}\right\rangle\left\langle p_{T M}|-| p_{C S}\right\rangle\left\langle p_{C S}\right|
\end{aligned}
$$

constructed on unit vectors written in the forms of (29)(32) and satisfying conditions (i)-(iii), with obvious symbol replacement.

It is then sufficient to show how we can construct the selfadjoint operator $\mathscr{E}_{A B}$. The remaining operators are obtained in the same way. An explicit construction was made in Aerts et al. (2019a). Repeating that construction for the data on the 'Google Images test' in Sect. 2, we find the following solutions.

We start by the joint measurement $e_{A B}$. We get:

$$
\begin{aligned}
& \left|p_{H G}\right\rangle=(0.99,-0.06,0.14,0) \quad \Theta_{H G}=145.80^{\circ} \\
& \left|p_{H W}\right\rangle=(0.02,0.94,0.30,0.14) \quad \Theta_{H W}=0.38^{\circ} \\
& \left|p_{B G}\right\rangle=(0.15,0.29,-0.94,0.04) \quad \Theta_{B G}=33.92^{\circ} \\
& \left|p_{B W}\right\rangle=(0.01,0.15,0,-0.99) \quad \Theta_{B W}=323.13^{\circ}
\end{aligned}
$$

We observe that the unit vectors $\left|p_{H G}\right\rangle$ and $\left|p_{B W}\right\rangle$ approximately represent product states, while the unit vectors $\left|p_{H W}\right\rangle$ and $\left|p_{B G}\right\rangle$ represent entangled states. Hence, $e_{A B}$ is an entangled measurement. 
Analogously, we can proceed to find solutions for the coincidence measurement $e_{A B^{\prime}}$, which are the following:

$$
\begin{aligned}
& \left|p_{H S}\right\rangle=(0.35,0.12,-0.93,0.05) \\
& \Theta_{H S}=0.07^{\circ} \\
& \left|p_{H M}\right\rangle=(0.01,0.33,0,-0.94) \\
& \Theta_{H M}=18.66^{\circ} \\
& \left|p_{B S}\right\rangle=(-0.03,0.94,0.13,0.33) \\
& \Theta_{B S}=69.29^{\circ} \\
& \left|p_{B M}\right\rangle=(0.94,-0.02,0.35,0) \\
& \Theta_{B M}=135.73^{\circ}
\end{aligned}
$$

We observe that the unit vectors $\left|p_{H M}\right\rangle$ and $\left|p_{B M}\right\rangle$ approximately represent product states, while the unit vectors $\left|p_{H S}\right\rangle$ and $\left|p_{B S}\right\rangle$ represent entangled states. Hence, $e_{A B^{\prime}}$ is an entangled measurement.

The solutions for the coincidence measurement $e_{A^{\prime} B}$ are instead the following:

$$
\begin{aligned}
& \left|p_{T G}\right\rangle=(0.51,0.54,-0.67,0.06) \\
& \Theta_{T G}=45.04^{\circ} \\
& \left|p_{T W}\right\rangle=(0.01,0.77,0.63,0.07) \\
& \Theta_{T W}=0.60^{\circ} \\
& \left|p_{C G}\right\rangle=(0.86,-0.33,0.39,0) \\
& \Theta_{C G}=12.97^{\circ} \\
& \left|p_{C W}\right\rangle=(0.03,0.08,0,-0.99) \\
& \Theta_{C W}=255.59^{\circ}
\end{aligned}
$$

We observe that all the unit vectors $\left|p_{T G}\right\rangle,\left|p_{T W}\right\rangle,\left|p_{C G}\right\rangle$, and $\left|p_{C W}\right\rangle$ represent entangled states. Hence, $e_{A^{\prime} B}$ is an entangled measurement.

Finally, the solutions for the coincidence measurement $e_{A^{\prime} B^{\prime}}$ are the following:

$$
\begin{aligned}
& \left|p_{T S}\right\rangle=(0.92,-0.20,0.33,0) \\
& \Theta_{T S}=354.14^{\circ} \\
& \left|p_{T M}\right\rangle=(0.06,0.30,0,-0.95) \\
& \Theta_{T M}=281.00^{\circ} \\
& \left|p_{C S}\right\rangle=(0.01,0.84,0.47,0.27) \\
& \Theta_{C S}=0.59^{\circ} \\
& \left|p_{C M}\right\rangle=(0.38,0.40,-0.82,0.15) \\
& \Theta_{C M}=45.06^{\circ}
\end{aligned}
$$

We observe that all the unit vectors $\left.\left|p_{T S}\right\rangle,\left|p_{T M}\right\rangle, p_{C S}\right\rangle$, and $\left|p_{C M}\right\rangle$ represent entangled states. Hence, $e_{A^{\prime} B^{\prime}}$ is an entangled measurement.

To conclude, we have proved in this section that the data collected in Aerts Arguëlles (2018) on visual perception exhibit the same type of strong entanglement that was identified in the psychological test in Aerts and Sozzo (2011) and Aerts et al. (2019a); namely, violation of the CHSH inequality is due to an entanglement that is present in both the initial state of the combined conceptual entity The Animal Acts and the coincidence measurements that are performed, due to the meaning connections between the component conceptual entities Animal and Acts.

\section{Entanglement as meaning connection}

Before concluding, it is worth to compare the violation of the CHSH inequality in visual perception (Aerts Arguëlles 2018) with the violation of the same inequality in the original psychological test on human participants (Aerts and Sozzo 2011).

Let us make more specific what we mean by considering the first joint experiment $A B$. In the psychological test, we asked the participants to choose among the four possible outcomes Horse Growls, Horse Whinnies, Bear Growls, and Bear Whinnies, and hence the data were collected after an interaction of the human minds of the participants with the questionnaire in the realm of a survey. Since the questionnaire contains a presentation of the experiment, the combination of concepts The Animal Acts is also present in some way in the realm of where the experiment takes place. However, we should not imagine that the state transformation modeled in Hilbert space in this paper, and also in Aerts et al. (2019a); Aerts and Sozzo (2014), describes a change of state in the mind of the participants. When we imagine from selfexperience what takes place in the minds of the participants, we would rather consider that the combination of concepts The Animal Acts is present in the overall realm of questionnaire mind, but not in such a way that each of the participants will undergo a transformation from the initial state describing Animal Acts to, for example, the state describing Horse Whinnies. Hence, we have to consider the state change modeled in Hilbert space and quantum collapse, as a "change of state of the considered concepts themselves'. This does not mean, however, that it is not interesting to connect what we can imagine to happen more specifically within the reality of the experiments, because this will reveal us reality aspects of the entities involved.

Let us hence turn in a more detailed way to how we imagine the experiment in the visual perception realm to be connected with the changes of states of the concept The Animal Acts to Horse Whinnies. In this experiment, there are no participants and minds of participants that interact within a questionnaire and no choosing of exemplars of the combination Animal Acts such as Horse Whinnies. However, the frequency of images of Horse Whinnies is counted, and after division by all the images considered and renormalization 
with the other three alternatives, we find a number which we consider to play the same role as the relative frequency of choices for Horse Whinnies in the psychological test. Why is it meaningful to do so? And, what insights does this give us to the connections it has with the state transformation modeled by the Hilbert space model?

The images which are shown when the search words 'horse whinnies' are entered in the 'Google Images' search engine are collected from the Web. Images on the Web are representative for images like they also are contained in human minds in imagination from experience and/or fantasy. Indeed, when an image containing Horse Whinnies is present in the 'Google Images' search, this means that this image was put on the Web by a human mind for a specific reason, which can be related to the reality of the person with this human mind, but also to the imagination of the person with this human mind. Hence, there is a less direct connection with the human mind in the experimental situation of the visual perception test considered in Aerts Arguëlles (2018) than in the experimental situation of the psychological test considered in Aerts and Sozzo (2011). The probabilities that are derived from the data are, however, in both cases equally well connected to the transformation of states and related probabilities of transformation as described in the Hilbert space model we presented in this article because of the way in which in both cases these probabilities are calculated.

We have already mentioned that the conceptual combination The Animal Acts can be imagined to be present in the questionnaire and its interaction with the human minds of the participants in the psychological test considered in Aerts and Sozzo (2011). But where is this combined concept The Animal Acts in the experimental situation in Aerts Arguëlles (2018) of the visual perception situation? All the images of 'Google Images' counted to give rise to the probabilities are images that contain more concrete animals, i.e., horses, bears, tigers or cats, performing more concrete acts, growling, whinnying, snorting or meowing, that the more abstract concepts Animal and Acts indicate. However, we can imagine a visual image where an abstract animal, hence a painting or drawing of an animal which cannot be recognized to be one of the concrete animals that we consider, so it could be a horse, a bear, a tiger or a cat, acts in a way that also cannot be distinguished between growling, whinnying, snorting, or meowing. This could, for example, be a painting or a drawing where the body and its parts of the animal depicted are very schematic, abstract, cubist, or impressionist, in such a way that no specific animal can be recognized, and the stature of this figurative animal painting or drawing is such that clearly a sound is made by the animal, while it is not possible to see in the painting or drawing which sound it is.

Can we claim in some way that the state transition described in the Hilbert space model is the more fundamental one and represents in a genuine way the underlying reality of what happens, both for the situation of the psychological experiment in Aerts and Sozzo (2011), and for which the Hilbert space model is worked out in Aerts and Sozzo (2014), and for the visual perception experiment in Aerts Arguëlles (2018)? We believe we can, and to bring forward part of the argumentation of why we believe we can, we want to do the opposite, and describe in some detail in which way the 'experimental happenings' taking place in both Aerts and Sozzo (2011) and Aerts Arguëlles (2018) are collapses or projections of the general transformation modeled in the Hilbert space formalism.

For the visual perception example considered in Aerts Arguëlles (2018) obviously, and actually as a consequence of the focus on visual perception, the 'presence of the considered animal in the three-dimensional theatre of space' is an important element of what is taking place. As mentioned already in Aerts Arguëlles (2018), the 'meaning content' of an image in a certain sense 'sticks out of the two dimensional plane in which the image is physically materially concepted as a consequence of its nature of being a visual imagine'. Of course, the 'sticking out of the plane' of the 'meaning content' does not have to be looked upon as if it sticks out in the third dimension of space; if that would be the case, it would not work any longer for a three-dimensional visual entity, such as a sculture. Hence, 'the meaning content sticks out of the plane' into another realm which is a 'meaning realm somewhere connecting the image with the human mind of the person watching it'. When we model this state of affairs and this type of dynamics quantum mechanically we inverse the view, i.e., the realm of meaning that we needed to introduce to be able to talk about what happens for the situation of visual perception, hence the realm of where the meaning content of the image sticks into, that realm is considered quantum mechanically to be the reality, and the material substance within the plane where the image is physically is a 'collapsed or projected state' of the real quantum mechanical state of the image, this real quantum mechanical state hence being non-spatial in nature, and referring to a state of being of this meaning realm. That is hence also how we have to interpret the state $p$ appearing in our Hilbert space model; it is the state Animal Acts referring to the non-spatial meaning realm in which the image sticks out to connect with the human mind of a person watching the image, this person's human mind equally so sticking out of the material collapsed state of his or her mind which is his or her brain.

We might believe that less of the above-described 'collapse or projection' is present in the case of the psychological test in Aerts and Sozzo (2011), but that is actually not really true. If we write the combination of concepts The Animal Acts, the written text is also a collapsed state of the concept. Also if we speak The Animal Acts, the sound of our speech taking place in time is a collapsed state of the con- 
cept. Both seem at first sight to be less intrinsically bound to space and/or time, but that is only appearance. Because of how language has evolved historically and also its purpose and how visual art has evolved and its purpose, we could believe that language is more faithful with respect to the structure of the reality of the meaning realm which we mention and which is non-spatial. This is partly true, but to put this in perspective, the way sentences are spoken and written is actually a collapse in a one-dimensional time-for the spoken version-and space-for the written versiondimension. That there is only one dimension available is also the reason that Animal and Acts are separately spoken and/or written, and joined only on the level of how the spoken and/or written form sticks out into the meaning realm they are collapses of. Because visual art makes use of three dimensions instead of the one dimension of spoken and written language, The Animal Acts will not have to be separated, and indeed, for the painting or drawing of The Animal Acts which we proposed above, its visual presentation does not separate Animal from Acts, both are joined, and the painting or drawing jointly represents, even in the collapsed state, Animal as well as Acts in one entity. Similarly for the different exemplars, we have considered in the experiments. Horse Whinnies in the one-dimensional collapse region of spoken and written language can only be represented by two separated pieces of spoken and/or written concepts, namely Horse and Whinnies, while in every image we see of a horse that whinnies, both Horse and Whinnies are joined inseparably within the collapsed form of the two-dimensional material of the image. For what concerns this important aspect of separation and non-separation, we could say that the visual art example of violation of the CHSH inequality might well be a better analogy for what happens in physics with the violation of these inequalities by means of entangled quantum entities. Indeed, one of the mysterious aspects of the entangled quantum entities is that the measuring apparatuses detecting the correlations can be widely separated in space, a quantum effect referred to as non-locality. In the way we have in our Brussels group analyzed the 'quantum entity situation of entanglement', we have often put forward the idea that the connection between both entities takes place in their non-spatial realms (Aerts 1990, 1998; Aerts and Sassoli de Bianchi 2016). In the two-dimensional collapsed state of their picture representation, Horse and Whinnies remain non-separated even in this collapsed state, while in the onedimensional collapsed state of their spoken and written word form, Horse and Whinnies are separated. This gives us a hint that quantum entities could well remain non-separated in their non-spatial states in which the entanglement takes place and only show themselves separated, like Horse and Whinnies do in their one-dimensional spoken and written collapsed state, in their three-dimensional space collapsed states, i.e., the regions of space where the detection apparatuses have been placed. Within our extended Bloch version of quantum theory, this notion of non-spatiality can be given a mathematical description as shown by the modeling of entanglement in this extended Bloch representation (Aerts and Sassoli de Bianchi 2016).

Hence, the entanglement encountered in physics including its manifestation of non-locality is perhaps not essentially different from the entanglement we have identified in cognition and visual art.

Acknowledgements This work was supported by QUARTZ (Quantum Information Access and Retrieval Theory), the Marie SkłodowskaCurie Innovative Training Network 721321 of the European Union's Horizon 2020 research and innovation program.

\section{Compliance with ethical standards}

Conflict of interest The authors have no conflict of interest to declare.

Human and animal rights This article does not contain any studies with human participants or animals performed by the author.

Open Access This article is licensed under a Creative Commons Attribution 4.0 International License, which permits use, sharing, adaptation, distribution and reproduction in any medium or format, as long as you give appropriate credit to the original author(s) and the source, provide a link to the Creative Commons licence, and indicate if changes were made. The images or other third party material in this article are included in the article's Creative Commons licence, unless indicated otherwise in a credit line to the material. If material is not included in the article's Creative Commons licence and your intended use is not permitted by statutory regulation or exceeds the permitted use, you will need to obtain permission directly from the copyright holder. To view a copy of this licence, visit http://creativecomm ons.org/licenses/by/4.0/.

\section{References}

Aerts D (1990) An attempt to imagine parts of the reality of the microworld. In: Mizerski J, Posiewnik A, Pykacz J, Zukowski M (eds) Problems in quantum physics. World Scientific, Singapore, pp 325

Aerts D (1998) The entity and modern physics: the creation-discovery view of reality. In: Castellani E (ed) Interpreting bodies: classi$\mathrm{cal}$ and quantum objects in modern physics. Princeton University Press, Princeton, pp 223-257

Aerts D (2009) Quantum structure in cognition. J Math Psychol 53:314348

Aerts Arguëlles J (2018) The heart of an image: quantum superposition and entanglement in visual perception. Found Sci 23:757-778

Aerts D, Sassoli de Bianchi M (2016) The extended Bloch representation of quantum mechanics. Explaining superposition, interference and entanglement. J Math Phys 57:122110

Aerts D, Sozzo S (2011) Quantum structure in cognition: Why and how concepts are entangled. Quantum Interaction. Lecture Notes in Computer Science 7052:116-127

Aerts D, Sozzo S (2014) Quantum entanglement in concept combinations. Int J Theor Phys 53:3587-3603

Aerts D, Aerts S, Broekaert J, Gabora L (2000) The violation of Bell inequalities in the macroworld. Found Phys 30:1387-1414 
Aerts D, Broekaert J, Gabora L, Sozzo S (2013) Quantum structure and human thought. Behav Brain Sci 36:274-276

Aerts D, Gabora L, Sozzo S (2013) Concepts and their dynamics: a quantum theoretic modeling of human thought. Top Cognit Sci 5:737-772

Aerts D, Haven E, Sozzo S (2018) A proposal to extend expected utility in a quantum probabilistic framework. Econ Theor 65:1079-1109

Aerts D, Beltran L, Geriente S, Sozzo S (2019) Quantum-theoretic modeling in computer science. A complex Hilbert space model for entangled concepts in corpuses of documents. Int $\mathbf{J}$ Theor Phys. https://doi.org/10.1007/s10773-019-04155-y

Aerts D, Aerts Arguëlles J, Beltran L, Geriente S, Sassoli de Bianchi M, Sozzo S, Veloz T (2019) Quantum entanglement in physical and cognitive systems: a conceptual analysis and a general representation. Eur Phys J Plus 134:493

Bell J (1987) Speakable and unspeakable in quantum mechanics. Cambridge University Press, Cambridge

Beltran L, Geriente S (2019) Quantum entanglement in corpuses of documents. Found Sci 24:227-246

Bruza P, Kitto K, Nelson D, McEvoy C (2009) Extracting spookyactivation-at-a-distance from considerations of entanglement. In: Bruza P, Sofge D, Lawless W, van Rijsbergen K and Klusch M (eds) Quantum Interaction. QI 2009. Lecture Notes in Computer Science 5494. Berlin, Heidelberg, pp 71-83

Bruza P, Kitto K, Ramm B, Sitbon L (2015) A probabilistic framework for analysing the compositionality of conceptual combinations. J Math Psychol 67:26-38
Gronchi G, Strambini E (2017) Quantum cognition and Bell's inequality: a model for probabilistic judgment bias. J Math Psychol 78:65-75

Busemeyer J, Bruza P (2012) Quantum models of cognition and decision. Cambridge University Press, Cambridge

Cirel'son BS (1980) Quantum generalizations of Bell's Inequality. Lett Math Phys 4:93-100

Clauser JF, Horne MA, Shimony A, Holt RA (1969) Proposed experiment to test local hidden-variable theories. Phys Rev Lett 23:880 884

Dalla Chiara ML, Giuntini R, Negri E (2015) A quantum approach to vagueness and to the semantics of music. Int J Theor Phys 54:45464556

Dalla Chiara ML, Giuntini R, Leporini R, Negri E, Sergioli G (2015) Quantum information, cognition, and music. Front Psychol 6:1583

Khrennikov A (2010) Ubiquitous quantum structure. Springer, Berlin

Melucci M (2015) Introduction to information retrieval and quantum mechanics. Springer, Berlin

Publisher's Note Springer Nature remains neutral with regard to jurisdictional claims in published maps and institutional affiliations. 